\title{
Voci, generi e spazi ibridi: La frontiera rovesciata di Francesco Burdin
}

Riassunto: Ne La frontiera rovesciata (1997) di Francesco Burdin, testo-collage di materiali eterogenei (con un saggio introduttivo, quattro canzoni e una cantata, tre racconti e un aforisma), si indaga intorno al concetto mutevole di frontiera con una vocazione ai cambi di prospettiva. In alcuni di questi testi, infatti, il discorso sulla frontiera e la narrazione intorno ad essa assieme agli effetti prodotti dalla guerra diventano predominanti e sembrano accogliere gli echi di alcune pagine di Italo Svevo e Karl Kraus sul primo conflitto mondiale. Ciascuno di essi si caratterizza, a sua volta, per la tendenza ad annullare i confini tra generi e forme, tra prese di parola individuali e collettive (e dunque con punti di vista differenti) e alla presenza di spazi storicamente connotati o in cui la natura sembra prendere il sopravvento rispetto alla logica bellica. In questo senso, l'effetto ultimo di queste due coordinate, la frontiera, intesa in termini concreti e metaforici, e la guerra (nelle sue ripercussioni e in alcuni scenari narrativamente codificati) sembrano farsi paradigma della condizione dell'uomo.

\section{Voices, genres, and hybrid spaces. Francesco Burdin's La frontiera rovesciata}

\begin{abstract}
In Francesco Burdin's La frontiera rovesciata (1997), a collection of heterogeneous materials (including an introductory essay, four songs and a cantata, three short stories and an aphorism), the shifting concept of border is investigated with a vocation for changing perspectives. In some of these texts, in fact, the discourse about the border and the narration around it pointing at the effects produced by the war become predominant and seem to echo some writings by Italo Svevo and Karl Kraus on the First World War. Each of the texts, in turn, is characterized by a tendency to blur the boundaries between genres and forms, between individual and collective perspective (therefore offering different points of view) and describe spaces which can be historically connoted or else pervaded by nature which seems to prevail over the logic of war. In this sense, the ultimate effect of these two coordinates - namely, the border, in concrete and metaphorical terms, and the war (with its repercussions and in some narratively codified scenarios) - seems to become a paradigm of the human condition.
\end{abstract}

2 Open Access. () 2020 Angela Fabris, published by De Gruyter. (c) BY-NC-ND This work is licensed under the Creative Commons Attribution-NonCommercial-NoDerivatives 4.0 License.

https://doi.org/10.1515/9783110640069-017 


\section{1}

In molte sue pagine Francesco Burdin, nato a Trieste nel 1916, indaga sul concetto mutevole di frontiera, con una vocazione al dubbio, all'interrogazione, ai cambi di prospettiva e all'esercizio dell'ironia. ${ }^{1} \mathrm{Ne}$ è un esempio illuminante il testocollage La frontiera rovesciata (1997), fondato sull'assemblaggio ben calibrato di materiali distinti: testi poetici, racconti, aforismi e un saggio introduttivo Viaggio a ritroso nel cuore della frontiera rovesciata - in cui l'autore si sofferma sulla natura fluida del varco che separa i due lati del confine. E dove l'io narrante appare proteso a recuperare un'identità che lo riporti alle origini della sua stirpe, all'interno di un territorio attraversato da confini labili, di natura e sostanza diversificata. Così, accanto a Cormòns («il luogo di origine della mia famiglia, sia dal lato paterno, sia da quello materno»), ${ }^{2}$ Burdin ricorda le esperienze e i vagabondaggi del nonno e del padre, e riflette sul rapporto con Trieste che assume il duplice significato di mito e realtà: «Ho lasciato Trieste all'età di nove anni, la triestinità è sopravvissuta integra nei decenni e anzi esaltata dall'esilio». ${ }^{3}$

Gilbert Bosetti sostiene che «l'esser triestini significa da sempre dubitare della propria identità». ${ }^{4}$ Ampliando l'asserzione, si può affermare che anche l'essere di frontiera induce a dubitare di essa. È il riflesso di un atteggiamento che Burdin intende in ottica ipotetica e costruttiva, alla luce di approcci e forme di riconoscimento variegate a cui si affiancano alcune considerazioni in merito agli scontri bellici e agli effetti da essi prodotti. Lo si evince dal discorso introduttivo con cui si apre il volume, Viaggio a ritroso nel cuore della frontiera rovesciata, la cui prima destinazione è stata un Convegno tenutosi a Grenoble il 4 novembre del 1994 e dedicato alla frontiera in tempi di guerra. Il discorso si sviluppa a partire da una precisa circostanza:

quando a mia insaputa, cambiai dal mattino al pomeriggio bandiera, sovrano, inno nazionale, corpus di leggi eccetera; insomma saltai, debole come ero a quell'età, le sbarre

1 Alcuni passaggi sono tratti da Angela Fabris, Un «de/scrittore» irriverente. Le strategie letterarie di Francesco Burdin, Pasian di Prato (UD), Campanotto, 2004. Diversi, tuttavia, sono l'oggetto dell'indagine e l'impostazione.

2 Francesco Burdin, Viaggio a ritroso nel cuore della frontiera rovesciata, in La frontiera rovesciata, Gorizia, Libreria Editrice Goriziana, 1997, p. 21.

3 Ivi, p. 11.

4 Gilbert Bosetti, Permanenza di una triestinità letteraria, in “Metodi \& Ricerche”, 19 (2002), n.1, pp. 109-134: 113. 
della frontiera. Anzi non ebbi nemmeno bisogno di saltare la frontiera: la frontiera si era rovesciata. ${ }^{5}$

Nel considerare la questione dei confini e l'improvviso ribaltamento dovuto alla prima guerra mondiale, un riferimento letterario che sorge spontaneo se si pensa al territorio goriziano e triestino del primo Novecento è l'immagine di Zeno in vacanza con la famiglia a Lucinico, sull'Isonzo, quando, senza rendersene conto, compie ripetuti sconfinamenti, ritrovandosi coinvolto - suo malgrado - nelle manovre preparatorie di quello che diverrà il fronte italo-asburgico; fino ad essere costretto a rinunciare non solo al caffellatte mattutino (privazione di cui si rammarica in più occasioni), ma anche a una prosecuzione della vacanza in quanto costretto a un frettoloso e solitario rientro a Trieste. Non a caso Brian Moloney - nel suo Italo Svevo narratore - considera la Coscienza nel suo insieme un romanzo di guerra, sottolineando come la reale cesura, dopo i sette capitoli in cui sono distribuite le memorie di Zeno, si produca allo scoppio del conflitto. ${ }^{6} \hat{\mathrm{E}}$ questo il momento in cui il narratore inizia ad assegnare una data esplicita alle sue memorie (a partire dal 3 maggio 1915), oltre a concedersi la sottile soddisfazione di inserire una fuorviante segnalazione temporale (il 23 maggio 1915) in cui Zeno respinge erroneamente, a più riprese, l'ipotesi di una dichiarazione di guerra da parte dell'Italia. A proposito del primo contatto di Zeno con la guerra scrive Cristina Benussi:

\footnotetext{
Svevo racconta con apparente distacco i momenti che precedettero l'aprirsi del fronte italoaustriaco, nella Coscienza di Zeno, anzi in quell'ultimo capitolo del romanzo, così diverso dagli altri. Qui il racconto infatti si trasforma in un diario che inizia il 3 maggio 1915, continua il 26 giugno 1915 e si conclude il 24 marzo 1916. È un capitolo fondamentale per dare un senso a quanto scritto in quelli precedenti, che raccontavano della cura intrapresa dallo psicanalista per guarire dal vizio del fumo. Dopo aver passato in rassegna tutti gli episodi fondamentali della sua vita [...] confermava, questa volta senza suscitare dramma, che in tutti i casi la molla ad agire era dettata dall'egoismo e dalla volontà di prevaricazione sugli altri. Inutile fingere di essere mossi da valori altruistici e nobilitanti, o da ritualità perbeniste civili e istituzionali, che in realtà coprono l'ipocrisia di fondo del patto sociale. È nell'ultimo capitolo infatti che la sua capacità ad adattarvisi rivela la sua salute rispetto alla malattia degli altri, scoprendo la vera natura dell'essere umano, e sua. ${ }^{7}$
}

5 F. Burdin, Viaggio a ritroso nel cuore della frontiera rovesciata, in La frontiera rovesciata, cit., p. 12.

6 Brian Moloney, La coscienza di Zeno come romanzo di guerra, in Italo Svevo narratore. Lezioni triestine, Gorizia, Libreria Editrice Goriziana, 1998, pp. 95-97.

7 Cristina Benussi, Confini. L'altra Italia, Brescia, Scholé, 2019, pp. 93-94. 
È l'ultimo capitolo, dunque, a chiarire il significato di quelli che precedono. Per quanto infatti la guerra si faccia notare fino a quel momento per la sua assenza, essa innerva il romanzo, ne costituisce un tacito perno. Nelle note pagine in cui lo sguardo di Zeno si sofferma su un plotone di soldati in marcia, quella che si può cogliere è una prima immagine del conflitto e soprattutto di una «nuova ed insuperabile frontiera ${ }^{8}$ che chiude il transito verso l'Italia.

Un'atmosfera in parte simile caratterizza il discorso introduttivo ne $L a$ frontiera rovesciata, a partire dalla constatazione che il concetto di patria, «sempre enigmatico» ${ }^{9}$ nei territori di frontiera, lo era ancor più nella compagine asburgica. Non a caso Burdin, in questo Viaggio a ritroso, osserva:

In un'Europa da settant'anni quasi tutta repubblicana è venuta meno la percezione del valore simbolico della regalità e, in particolare, della funzione connettiva della monarchia asburgica. Ma senza di essa non si può capire l'essenza stessa, lo spirito, dell'impero [...]. E la persona fisica di Francesco Giuseppe, che per sessantotto anni offre una quasi ottusa dedizione al suo ruolo, pareva pietrificata apposta per mantenere inalterabile la fedeltà e la fiducia nei suoi sudditi. Nessuno dei quali al principio del secolo poteva immaginare così prossima l'estinzione dell'impero millenario. ${ }^{10}$

In quest'ottica la ricostruzione della memoria biografico-familiare e il ripercorrere con spirito disincantato il mito delle origini passa attraverso l'inizio della prima guerra mondiale: «Per le strade di Trieste nemmeno un'oncia di quell'entusiasmo guerresco prossimo all'isteria che vediamo testimoniato dalle foto d'epoca». ${ }^{11}$ La guerra, fin da subito «feroce», diviene anche lo spunto per tessere un mosaico di richiami letterari, da Joseph Roth a Georg Trakl fino a Slataper e Stuparich, dei quali scrive che da Trieste, dopo aver passato le linee, «andarono a morire in uniforme grigioverde, sotto falso nome». ${ }^{12}$

Burdin viene poi a concludere il suo excursus su Trieste affermando: «Quanto a Trieste, oggi che la frontiera si è rovesciata, è davvero una città di frontiera: lo era meno nel 1914, quando le sbarre di confine erano distanti sessanta chilometri». ${ }^{13}$

8 Italo Svevo, La coscienza di Zeno, a cura di Beatrice Stasi, Roma, Edizioni di Storia e Letteratura (Edizione Nazionale dell'Opera Omnia di Italo Svevo), 2008, p. 412.

9 F. Burdin, Viaggio a ritroso nel cuore della frontiera rovesciata, in La frontiera rovesciata, cit., p. 11.

10 Ivi, p. 16.

11 Ivi, p. 18.

12 Ivi, p. 20.

13 Ivi, p. 21. 
Anche Cormòns riveste un ruolo specifico sul piano familiare (lo si è detto), su quello letterario (nel 1582 vi nasce «Ludovico Lepòreo, poeta barocco di cui sono memorabili le bizzarrie inventive: dunque una specie di Arcimboldo della penna. Qualità dopo tutto anche a me imputate») $)^{14}$ e in termini di confine: «Nel 1914 Cormòns era da quattrocento anni l'avamposto del confine orientale austriaco: cinque chilometri più a ovest il fiume Judrio era la frontiera. Dopo veniva la terra di San Marco, l'Italia». ${ }^{15}$

\section{2}

Concluso quello che definisce un "discorso sulle patrie in terra di confine», ${ }^{16}$ Burdin cede spazio a materiali distinti, tra cui due racconti che affrontano situazioni narrativamente codificate della vita militare, quali il riconoscimento del nemico come fratello e il dopo battaglia. Nel primo di essi, L'ordine naturale, tenta di sondare lo spazio delle possibilità che si aprono all'uomo, combattuto tra il senso del dovere e il rispetto della natura umana, fra le logiche della guerra e l'istinto naturale, fra la vita e la morte. Lo si evince anche dall'epigrafe che riporta i versi di Carlo Michelstaedter tratti da Il canto delle crisalidi (1909): «Vita morte, / la vita nella morte; / morte vita, / la morte nella vita». ${ }^{17}$

Il protagonista è «un soldato in servizio notturno di sentinella a un osservatorio dell'artiglieria, in montagna». ${ }^{18} \mathrm{Al}$ centro del percorso si situano le riflessioni di questo giovane non ancora ventenne, cresciuto in un orfanotrofio, che si ritrova improvvisamente in mezzo alla vita militare nel corso della prima guerra mondiale. Partendo dal presupposto che l'uomo è l'unico essere in grado di progredire, la recluta si convince che il destino dell'individuo sia quello di opporsi e non di assoggettarsi all'ordine naturale. Soprattutto in un universo - il nostro - nel quale l'orizzonte di attese dell'uomo si è drammaticamente ridotto: «Ma quanto al futuro, che cosa mi aspetta? I conti non sono difficili. Lavorerò

14 Ivi, p. 22. Per uno sguardo d'insieme sull'opera di Francesco Burdin vedi Cristina Benussi, Francesco Burdin, in «Berlfagor», 45 (1990), n. 3, pp. 293-306 e Gilbert Bosetti, Les impertinences de Francesco Burdin entre humour et auto-ironie, «Italies, Revue d'études italiennes», Université de Provence, 4/2 (2000), pp. 633-646 (consultabile anche online: http://journals.openedition. org/italies/2357 ; DOI : https://doi.org/10.4000/italies.2357).

15 Ibid.

16 Ivi, p. 26.

17 F. Burdin, L'ordine naturale, in La frontiera rovesciata, cit., p. 71.

18 Ibid. 
dieci ore sotto padrone, avrò, se mi tocca, moglie e figli da sfamare, aspetterò la domenica e le altre feste comandate per passarle davanti a una tavola di osteria». ${ }^{19}$

Un futuro già scritto cui decide di opporsi. Il quesito è quale debba o possa essere l'atto con cui sottrarsi ad esso; forse quello di tendere la mano al nemico che si arrampica verso di lui, sulla scia di un impulso che lo porta alla morte:

«Bella notte!» egli sussurra. Sente battere il cuore per il timore che l'‘altro' venga tentato dal suo medesimo proposito. Il freddo della lama che lo penetra nel ventre gli fa intendere che non è così.

Il nemico ha tenuto fede all'ordine naturale..$^{20}$

La vicenda si focalizza sul confronto tra l'istinto di solidarietà e la logica negativa, alimentata dai doveri e dagli obblighi imposti dalla guerra, a cui deve sottostare il soldato. Non a caso la recluta sottolinea sarcasticamente l'assurdità della vita militare tramite verità scorciate o assunti di natura aforistica: «Il comico esiste nella vita di tutti i giorni, il servizio militare ne è un esempio». ${ }^{21}$ Ancora: «Nessuno ha mai preteso che la guerra sia confortevole».22

Altrove, nel racconto, a prevalere sono invece passaggi contraddistinti da un moto riflessivo:

Mai la volta stellata gli è apparsa tanto profonda, gli astri così enormi e luminosi. Li ha osservati molte notti nei servizi di guardia: forse i suoi pensieri andavano altrove, o più semplicemente non aveva pensieri. Del resto anche di giorno non vi è mai necessità né occasione di riflettere. ${ }^{23}$

Anche in questo passaggio è evidente una misura aforistica; infatti, se isolato dal contesto non subisce una riduzione di significato, nella collocazione originaria accentua invece il carattere paradigmatico della vicenda. È una tendenza visibile anche in alcuni quesiti di natura conoscitiva:

Dovere e potere che cosa sono? Non sono oggetti come il gong e il fucile. Direi, se devo trovare un loro titolo, che sono pure invenzioni della società civile, tanto quanto i Dieci comandamenti lo sono di quella religiosa. ${ }^{24}$

19 Ivi, pp. 81-82.

20 Ivi, p. 88.

21 Ivi, p. 74.

22 Ivi, p. 73.

23 Ivi, p. 72.

24 Ivi, p. 85. 
In questo caso, ripristinando la frase nella sua interezza il narratore si affida a una sorta di lucido raziocinio. Il racconto si caratterizza così sia per il suo circoscrivere a livello tematico specifiche zone di confine (tra la logica bellica e l'ordine naturale), sia per la scrittura che accoglie al suo interno i confini, mutevoli e a volte sfuggenti, di una prosa che dalla descrizione si apre ad un sottile tenore riflessivo per poi cedere al cortocircuito dell'aforisma o ancora a un registro espressivo contratto.

Il dopo battaglia è invece al centro del racconto Il generale nel bosco in cui nell'esordio - si assiste alla descrizione del processo che dallo scontro tra «due uomini soli $»^{25}$ conduce a un conflitto più ampio:

Delle battaglie di terra non è mai stato facile determinare, a cose fatte, l'attimo dell'inizio, quasi che anche qui confluissero - come per i momenti storici - più cause e svariate circostanze puramente occasionali.

I primi contatti sono per lo più tra due uomini soli che si scontrano e si sparano a bruciapelo senza aver tempo di domandarsi i documenti [...]. Sempre senza conoscersi - a che varrebbe fra uguali? - si sventrano, si fulminano e si intossicano con le armi meglio perfezionate che uomini più esperti e maturi hanno fornito [...]. Finalmente si stendono placati, con l'ultimo terrore disegnato nell'angolo della bocca, e dentro gli occhi, se frughi anche senza particolare intenzione, una intensa sete di requie e di ritorno, conseguita a caro prezzo quella, irraggiungibile il secondo. Perché veramente dalla battaglia non ritorna nessuno. Tutti siamo rimasti lì. ${ }^{26}$

Il periodo finale segna il passaggio da una prospettiva esterna a una interna, corale e partecipativa, di carattere aforistico. È quanto accade anche nel progredire del racconto in cui si assiste all'irruzione di immagini metaforicamente in grado di esprimere le connotazioni psicologiche del dopo battaglia: «la terra esplodeva con le nostre anime contro il cielo», ${ }^{27}$ «non vi era più alcuno delle migliaia e migliaia che il giorno prima erano là presenti con l'anima e la paura». ${ }^{28}$

Il bosco, superato l'orrore dei combattimenti e lavato dall'acqua del violento temporale che vi segue, pare acquisire una sua natura antropomorfa, con i tronchi che «si appoggiavano spalla contro spalla, riparando le ferite». ${ }^{29}$ Una natura che viene, in tal modo, ad annullare le fratture e le lacerazioni, assieme ai confini tracciati dagli scontri:

25 F. Burdin, Il generale nel bosco, in La frontiera rovesciata, cit., p. 121.

26 Ivi, pp. 121-122.

27 Ivi, p. 122.

28 Ivi, p. 123.

29 Ibid. 
Sebbene provata dai disordini della battaglia, la foresta appariva al cielo compatta e integra. Il verde era colorito dall'acqua, i sentieri che avevano conosciuto la vigilia tanta animazione erano invisibili sotto le fronde eccitate e gli arbusti vivificati. ${ }^{30}$

Quello che era stato lo scenario della battaglia, uno «spazio striato» con sentieri e recinti, si trasforma nuovamente, sulla scia di Deleuze e Guattari, in uno spazio nomade «liscio, marcato soltanto da 'tratti' che si cancellano e si spostano lungo il tragitto», ${ }^{31}$ fino a divenire un «bosco senza limite né fine». ${ }^{32}$ Non a caso, della dimensione bellica rimane traccia solo nella marcia del generale e dei due subalterni, colti nel loro disperato procedere di superstiti, «il passo e lo sguardo privi di intelligenza». ${ }^{33}$

Inframmezzate alle osservazioni sulla natura che riprende il sopravvento, trovano posto le pacate osservazioni del narratore nei confronti dei soldati e della loro angoscia:

uomini del nord, del sud, alti e denutriti delle fabbriche, piccoli e scuri cresciuti tra il granoturco e l'olivo, reclute o richiamati di mezzo secolo, con gli occhi dilatati dal caldo e dalla marcia, gente fatta per buttare il fucile non per attaccare. Migliaia e migliaia di esseri, carne e respiro, erano così assenti come non fossero mai esistiti. ${ }^{34}$

Di seguito si sviluppa - dapprima quasi inavvertitamente, poi in un crescendo di intensità - un implicito confronto tra una disciplina gerarchicamente intesa, con le disamine in un primo momento boriose e successivamente angosciate del generale, e il riapparire ad intermittenza di un coro a più voci, quello dei soldati periti nel massacro.

Le considerazioni del generale sono caratterizzate e sostenute dal fitto ricorrere di punti esclamativi a siglare frasi colme di rabbia - «Nessuno può dire che io abbia sbagliato [...]. Il congegno era perfetto, niente lacune, nessun punto debole, niente di imprevisto! $»^{35}$ - o punti di domanda irati - «Dove sono finiti quei maledetti che ieri a quest'ora, nell'obbligo del silenzio, si preparavano

30 Ivi, p. 125.

31 Gilles Deleuze e Félix Guattari, Millepiani. Capitalismo e schizofrenia, trad. it. di Giorgio Passarone, Roma, Castelvecchi, 1987, p. 557.

32 F. Burdin, Il generale nel bosco, in La frontiera rovesciata, cit., p. 127.

33 Ivi, pp. 123-124.

34 Ivi, pp. 124-125.

35 Ivi, p. 124. 
divorando all'assalto?». ${ }^{36}$ Il tutto intervallato da quesiti densi di stupore - «Che fanno lassù?» ${ }^{37}$ - o dal ripercorrere mentalmente gli ordini impartiti:

il fronte non si spezza. Via questi imbecilli, feriti senza necessità, morti senza merito, spazzate il terreno. Dio come è lunga questa faccenda. Fra poco sarà il diluvio. Che cosa? Sparate sulla schiena, sparate senza misericordia anche sugli ufficiali. Non voglio nessuno indietro dalla linea del fuoco. I feriti se li tengano. Sparate. Se uno si ritira, tutti indietreggiano. La mia divisione non crolla. Io vi fucilo tutti. ${ }^{38}$

Alle disamine del generale si oppone il sogno-visione del tragico coro dei soldati, incapaci - a detta del superiore - di esprimere soddisfazione per una fine onorevole; al contrario, le loro voci atterrite sono colme di lamenti e quesiti sarcastici intrisi di amarezza:

- Noi facciamo ancora la nostra figura, generale?

- Guarda come stiamo impettiti sull'attenti, le nostre uniformi sono candide più del vento.

- E io, io troppo stupido per capire la guerra?

- Ne sappiamo ormai di più sugli attacchi a massa, sugli aggiramenti e lo sfruttamento del successo. ${ }^{39}$

Sono le battute di un dialogo che si protrae per più pagine, e che sottopone a un rovesciamento di prospettiva il ruolo del nemico, fino a coglierne il comune e tragico destino («non è cattivo il nemico; è vero, è vero, è vero; geme e striscia con noi il nostro nemico, generale»). ${ }^{40}$

Non manca la descrizione dell'attesa carica di angoscia prima dell'attacco:

Io non volevo uscire da dietro il tronco. Mi pareva così accovacciato, la bocca tuffata contro il muschio, di trovarmi in un luogo chiuso, in un orribile agguato, nell'attesa di qualcuno mai visto, che sarebbe venuto per trucidarmi. E io lì, col cuore in gola, a tormentare il fucile, raffigurandomi lo spaventoso viso di questo ignoto omicida, e domandandomi perché..$^{41}$ Io non volevo uscire da dietro al tronco. Mi hanno spinto con un calcio e sono andato a morire due metri più in là. Non ho fatto una gran strada. ${ }^{42}$

36 Ibid.

37 Ivi, p. 125.

38 Ibid.

39 Ivi, p. 129.

40 Ivi, p. 130.

41 Ibid.

42 Ivi, p. 131. 
Vi si accompagna un senso di inutilità: «La gloria, generale, la gloria non è per i soldati. Chi poteva mai prendere quel colle?».43

Il tragico coro segue il generale passo dopo passo; le recriminazioni finiscono per acuirne la rabbia e la sensazione di colpevolezza che egli si sforza inutilmente - di reprimere: «Orbene, eccellenze, l'esercito è scomparso, volatilizzato come un arrosto bruciato. Trentamila uomini perduti. Errore di calcolo, ostinazione? no, un generale non fa errori di calcolo e non è ostinato, se mai tenace. Tradimento!». ${ }^{44}$ Fino a denunciare tramite un cambio di registro con il passaggio ad una serie di inserzioni di natura quasi teatrale nel loro andamento dialogico e a tratti monologante - il chiaro processo di automistificazione posto in atto da chi, come lui, cerca di riproporre come necessari e onorevoli le scelte e gli atti compiuti.

La tensione giunge al culmine e si stempera in amarezza nella pagina finale, dove si assiste ad un accentuarsi dell'aspetto paradigmatico della vicenda tramite l'immagine di un cane, «una bestia da battaglione, col pelo chiazzato e una gran fame negli occhi», ${ }^{45}$ impaziente di leccare le ferite del generale in agonia. La logica della natura contrapposta ai meccanismi distorti della guerra, fondati su una dinamica distruttiva. E una zona di confine tra la disciplina, le disamine supponenti del generale e il sogno-visione di un tragico coro che rende ragione di quanto asserito da Holger Klein, secondo il quale le narrazioni di guerra devono essere intese non tanto o non solo come la descrizione ipotetica di uno specifico avvenimento od episodio, quanto in merito agli effetti prodotti e alle ricadute sull'esistenza degli individui. ${ }^{46}$ Lo attesta, nella sua drammaticità, questo racconto di Burdin, sulla scia di una dimensione corale che acquista i tratti di un tragico atto recriminatorio.

\section{3}

Il riaffiorare persistente di un molteplice e caotico insieme di voci intorno e sulla prima guerra mondiale è anche al centro della «tragedia in cinque atti» di Karl

43 Ibid.

44 Ivi, p. 132.

45 Ibid.

46 Cfr. Holger Klein (a cura di), The Second World War in Fiction, London, MacMillan, 1984, p. 12. Nell'introduzione al volume, che è una raccolta di saggi sulla narrativa di finzione della seconda guerra mondiale, il curatore riflette intorno alla sostanza ed essenza di un romanzo di guerra. 
Kraus, Die letzten Tage der Menschheit, in italiano Gli ultimi giorni dell'umanità. Testo straripante, sorto in parallelo ai drammatici sviluppi della guerra, appare sulla scena letteraria, in una prima veste editoriale, nel 1919, per essere poi destinato alle stampe nella sua versione definitiva nel 1922 e successivamente nel 1926. Nonostante l'impianto teatrale, il testo non può essere rappresentato nella sua totalità a causa dell'alternarsi di circa cinquecento personaggi e dell'affastellarsi di un ammasso di voci, singole e plurime. È una serie di battute che si alternano a due, a tre o più, nel succedersi e ripetersi spesso volutamente monotono di oltre duecento scene. Non a caso il medesimo Kraus si limitò a darne alcune letture pubbliche dopo aver chiarito, nella Premessa, le ragioni di questa scelta:

Theatergänger dieser Welt vermöchten ihm nicht standzuhalten. Denn es ist Blut von ihrem Blute und der Inhalt ist von dem Inhalt der unwirklichen, undenkbaren, keinem wachen Sinn erreichbaren, keiner Erinnerung zugänglichen und nur in blutigem Traum verwahrten Jahre, da Operettenfiguren die Tragödie der Menschheit spielten. ${ }^{47}$

I frequentatori dei teatri di questo mondo non saprebbero reggervi. Perché è sangue del loro sangue e sostanza della sostanza di quegli anni irreali, inconcepibili, irraggiungibili da qualsiasi vigile intelletto, inaccessibili a qualsiasi ricordo e conservati soltanto in un sogno cruento, di quegli anni in cui i personaggi da operetta recitarono la tragedia dell'umanità. ${ }^{48}$

Il testo si dipana tra luoghi differenti in primis della realtà viennese: il viale del Ring, il Caffè Pucher, la stazione Sud, la cancelleria del Ministero della Real Casa fino agli angoli di strada, colti nel continuo formarsi di capannelli e nelle illogiche reazioni della folla. A queste ambientazioni cittadine si mescolano altri scenari, inclusi alcuni quadri di guerra; per esempio, il fronte della Bucovina, quello sud-occidentale o i campi di battaglia. Il testo, straripante nel delirio allucinatorio di un composito insieme di personaggi di cui è impossibile dar conto, si dispone in una struttura a stazioni, con ritorni ai medesimi luoghi e personaggi e al vuoto cicaleccio che li caratterizza. ${ }^{49}$

47 Karl Kraus, Die letzten Tage der Menschheit, https://www.projekt-gutenberg.org/kraus/ letzttag/chap001.html (consultato il 20.8.2020).

48 Karl Kraus, Gli ultimi giorni dell'umanità. Tragedia in cinque atti con preludio ed epilogo, edizione italiana a cura di Ernesto Braun e Mario Carpitella, con un saggio di Roberto Calasso, Milano, Adelphi, 1980, p. 9.

49 Daniel Weidner, Weltkriegstheater. Botenbericht und Mauerschau in Karl Kraus' Die letzten Tage der Menschheit, in Kriegstheater. Darstellungen von Krieg, Kampf und Schlacht in Drama und Theater seit der Antike, a cura di Michel Auer e Claude Haas, con la collaborazioe di Gwendolin Engels, Stuttgart, J. B. Metzler, 2018, pp. 247-260. 
In tale variegato concorrere di voci e figure allusive della scena viennese dell'epoca, quello a cui si assiste è una sorta di processo di disgregazione dell'uomo, dissolto in una miriade di frammenti verbali. Sono scambi privi di significato quelli che caratterizzano questi «personaggi da operetta» che si contraddistinguono, in alcuni casi, per il loro essere radicati nella dimensione storica, politica e sociale dell'epoca o per rappresentare la folla nelle sue non meditate reazioni. È evidente la capacità di racchiudere in una rete di parole vacue e destituite di senso gli scambi dialogici o gli interventi degli ufficiali bellimbusti, dell'abbonato, del giornalista, del politico, dello strillone, della massaia patriottica, dell'ubriaco, dei cerimonieri di Corte, dei commercianti e dei poeti fino ai due imperatori, Francesco Giuseppe e Guglielmo II, con le loro voci illustri, alternate ad altre che recano in sé i tratti dell'anonimato. Si assiste così ad un ininterrotto delirio allucinatorio che permette una complessa raffigurazione - principalmente in via indiretta - della guerra. Al riguardo acquista peso la figura della Schalek, ${ }^{50}$ reporter dalla moralità altalenante che visita le trincee in cui sono impiegati i soldati dell'Impero asburgico, e che, nel descrivere quanto vede, costruisce una serie inesauribile di verità distorte, sostenuta dall'esaltazione delle armi e dalla logica della guerra a cui aderisce con entusiasmo. Accanto, quali sporadiche apparizioni, sfilano non solo coloro che la guerra l'hanno voluta ma anche gli speculatori che, grazie a essa, si sono arricchiti, i patrioti, i fornitori militari, i corrispondenti di guerra o i pavidi piccolo-borghesi costretti alla cautela e all'obbedienza. E non mancano, nella scena cinquantacinquesima del quinto e ultimo atto, le voci dei soldati assiderati sui Carpazi:

Bei der vordersten Linie in den Karpathen. Es ist alles ruhig. In den Schützengräben stehende Leichname. Mann neben Mann, das Gewehr im Anschlag.

Die erfrorenen Soldaten:

Kalt war die Nacht.

Wer hat diesen Tod erdacht!

Oh die ihr schlieft in Betten -

daß euch das Herz nicht bricht!

Die kalten Sterne retten uns nicht.

50 Vedi Elisabeth Klaus, Rhetoriken über Krieg: Karl Kraus gegen Alice Schalek, «Feministische Studien», Vol. 26 (2008), n. 1, pp. 65-82 e Mira Miladinović Zalaznik, Die Reporterin Alice Schalek bei der Isonzoarmee, «Zagreber Germanistische Beiträge, 25 (2016), pp. 271-290. 
Und nichts wird euch erretten! ${ }^{51}$

In prima linea sui Carpazi. Tutto tace. Cadaveri in piedi nelle trincee, l'uno accanto all'altro, col fucile puntato.

I soldati assiderati

La notte era gelata.

Questa morte, chi l'ha inventata!

Oh voi che a letto dormivate -

Il cuor non vi si spezza?

Le fredde stelle non ci dan salvezza,

e anche voi, di salvarvi non sperate $!^{52}$

L'insieme multiforme di voci che Kraus raccoglie non è solamente integrato nell'azione drammatica della tragedia - quasi una sorta di mormorio relegato sullo sfondo - ma è la tragedia stessa. Ed è dunque questo insieme di parole nelle loro moltiplicazioni acustiche - che consente di approdare a una complessa rappresentazione della Grande Guerra a cui si affianca significativamente la presenza di due personaggi-guida - l'Ottimista e il Criticone - che, in veste dialogica, ottemperano alle funzioni di una sorta di coro, dando voce ad istanze divergenti. Sono battute e scambi dialogici che, oltre ad assolvere a una funzione ordinatrice, esprimono posizioni distinte in chiave esplicativa e dove il Criticone commenta quanto accade dentro e fuori dei campi di battaglia (tra sé o interloquendo) e si fa portavoce della caustica opinione di Kraus di cui il personaggio è proiezione letteraria.

È a lui, al Criticone, che si deve ricondurre il manifesto di congedo con cui prende avvio la scena 54, dove egli appare per l'ultima volta, in un drammatico e struggente monologo:

Ich habe eine Tragödie geschrieben, deren untergehender Held die Menschheit ist; deren tragischer Konflikt als der der Welt mit der Natur tödlich endet..$^{53}$

Ho scritto una tragedia il cui eroe soccombente è l'umanità; il cui conflitto tragico, essendo quello tra mondo e natura, finisce con la morte. ${ }^{54}$

51 K. Kraus, Die letzten Tage der Menschheit, https://www.projekt-gutenberg.org/kraus/ letzttag/chap007.html (consultato il 20.8.2020).

52 K. Kraus, Gli ultimi giorni dell'umanità, cit., pp. 652-653.

53 K. Kraus, Die letzten Tage der Menschheit, https://www.projekt-gutenberg.org/kraus/ letzttag/chap007.html (consultato il 20.8.2020).

54 K. Kraus, Gli ultimi giorni dell'umanità, cit., Scena cinquantaquattresima. Il criticone alla scrivania. Legge, p. 608. 
Nel finale, lo straripante testo di Kraus si chiude sotto il segno di una serie di visioni che si succedono le une alle altre con vittime che prendono brevemente la parola (Scena cinquantacinquesima). L'epilogo, infine, risulta assemblato intorno a brevi strofe in rima in cui intervengono i carnefici (per esempio il generale $\mathrm{e}^{55} \mathrm{ole}$ iene con il loro valzer intorno ai cadaveri) ${ }^{56}$ e a cui si contrappone il soldato morente che grida assieme a una profusione di voci (la prima, la seconda, la terza, la quarta, queste e quelle, l'una e l'altra, tutt'e due $)^{57}$ e a cui si affianca un oggetto simbolo, la maschera antigas, che pare alludere al destino che attende l'uomo travolto dalla tragicità del primo conflitto mondiale. Al di sopra di questo scenario apocalittico, dopo un «immane silenzio», ${ }^{58}$ si ode una voce dall'alto - la voce di Dio - che riprende le famose parole di Guglielmo II, pronunciate nel 1915 durante la visita a un campo di battaglia sul fronte francese: «Io non l'ho voluto». 59

\section{4}

Il coro di voci di Kraus si posiziona dunque agli antipodi rispetto a una concezione della guerra quale strumento di rigenerazione. Sul piano individuale è quanto accade nella Cantata del vecchio Franzil, dove il padre di Burdin, in versi sciolti e in prima persona, rievoca la dinastia familiare, la giovinezza, le varie peregrinazioni ed esperienze, inclusa la guerra:

\footnotetext{
a ventun anni per ordine del Kaiser

andai a cavalcar con brache rosse a Graz

el Zugfier comandava schnell in tedesco, a

ventiquattro marciai sei dì col novanta

sette di fanteria di linea alla frontiera

e i paesani mi chiamavano in polacco

la pallottola russa a Grodek, Galizia,

mi bucò un braccio. ${ }^{60}$
}

55 Ivi, p. 662.

56 Ivi, p. 679.

57 Ivi, pp. 682-685.

58 Ivi, Epilogo, p. 692.

59 Ibid. Nell'originale: «Ich habe es nicht gewollt», https://www.projekt-gutenberg.org/kraus/ letzttag/chap008.html (consultato il 20.8.2020).

60 F. Burdin, Cantata del vecchi Franzil, in La frontiera rovesciata, cit., 54. 
In questo universo mosso e accidentato i cambi di scena sono direttamente proporzionali alle lingue che vi riecheggiano (tra friulano, sloveno, croato, tedesco e dialetto triestino), in un testo articolato in sette strofe in cui il concetto di frontiera è sottoposto a continui rovesci e scossoni. E nel quale, nell'ultima strofa, dopo aver considerato quanto gli è stato concesso e poi sottratto dall’Impero, Franz ragiona con lucidità e osserva:

\section{[...] A}

parer mio si cambian le bandiere

ma il confine tra quel che va e quel che

non va nel mondo non è

poi così stabile, le differenze

son piuttosto apparenze,

la sorte di chi vien fuori da una pancia

è quella, che

l’ordine vi sia oppure no è trascurabile. ${ }^{61}$

Conclusione che rinvia ad una sorta di azzeramento delle frontiere geopolitiche al di là dei cambi di potere in una ballata dotata di una certa eleganza ritmica. La Cantata (al pari delle quattro canzoni raccolte in questa sezione de La frontiera rovesciata) è preceduta da un inserto intitolato Per la satira anche il narratore sceglie talvolta il verso, in cui Burdin spiega le ragioni del cambio di «attrezzeria»:

accade (è accaduto) al narratore, incline all'ironia, ma desideroso di impegnarsi più a fondo nella satira, di trovare necessari alcuni strumenti che mancano o sono limitati nel suo armamentario: per esempio una corda più alta e più secca, un linguaggio più penetrante $\mathrm{e}$ corrosivo, un taglio che non lasci spazio a intrugli retorici o sentimentali, e, pronti, a portata di mano, invenzioni, paradossi e capricci dell'immaginazione. ${ }^{62}$

In effetti, sia in questo testo che nel saggio introduttivo Viaggio a ritroso nel cuore della frontiera rovesciata e nei due racconti analizzati, assieme agli echi letterari di autori immersi nell'atmosfera primonovecentesca dell'Impero (Svevo, Kraus e Michelstaedter) si assiste, di continuo, al variare di generi, piani e registri assieme all'intrecciarsi delle implicazioni spazio-temporali della frontiera con le disamine negative intorno alla guerra. Nella ricostruzione delle coordinate biografiche e familiari, nei tracciati narrativi o nella Cantata si delinea, in forma più o meno implicita, una sorta di negazione nei confronti di un concetto univoco di appartenenza a vantaggio di una rete di commistioni identitarie, linguistiche, culturali e letterarie.

61 Ivi, p. 63.

62 Ivi, p. 30. 
Anche lo spazio riveste un suo ruolo, tra Trieste e Cormòns dove si riattiva il mito delle origini e la memoria familiare, i territori dell'ex-Impero in cui si muove girovagando Franzil ed altri spazi oggettivamente connotati; è il caso della foresta che inghiotte e cancella la battaglia, cui si aggiunge il situarsi e riconoscersi della sentinella in un perimetro geografico - il fronte - sottratto nella sua percezione a interferenze politiche: «da diciotto mesi gli appartengono il respiro dell'aria libera, lo splendore diurno della grande vallata, la successione dei monti che si susseguono senza fine; il verde dei boschi lontani, il passaggio a ondate delle nuvole». ${ }^{63}$

Ciascuno di questi testi, al suo interno, riflette il coesistere di forme e generi differenti, tra la divagazione memoriale o saggistica, il verso o la narrazione, e tra la misura scorciata di una verità bruciante e le variazioni poetiche e irriverenti di una sorta di moderno picaro fino alla presenza di un coro di voci; forme ibride che si caratterizzano per l'alternarsi di generi differenti, di spazi (concreti e astratti, reali e immaginari, assieme al dato oggettivo e a quello ipotetico), con una scrittura umorale in cui piani e registri si intersecano senza sosta assieme all'alternarsi di prospettive individuali e collettive.

Mentre Zeno personifica l'egoismo e la ritrovata salute di fronte a un conflitto europeo e il Criticone di Kraus si congeda drammaticamente, la sentinella di Burdin sceglie di morire per sfiducia nel futuro e il generale si spegne sopraffatto da una natura volta a farsi nuovamente «spazio liscio». La guerra - e con essa la frontiera - diviene paradigma della condizione dell'uomo.

63 Ivi, p. 73. 